\title{
Carbon Monoxide and Stroke: a Time Series Study of Ambient Air Pollution and Emergency Hospitalizations
}

Linwei Tian ${ }^{1 *}$, Hong Qiu ${ }^{1}$, Vivian C. Pun ${ }^{2}$, Kin-Fai Ho ${ }^{2}$, Chi Sing Chan ${ }^{2}$, Ignatius T.S. Yu ${ }^{2}$

\section{Authors' institutional affiliations:}

${ }^{1}$ : School of Public Health, Li Ka Shing Faculty of Medicine, The University of Hong Kong; ${ }^{2}$ : The Jockey Club School of Public Health and Primary Care, The Chinese University of Hong Kong, Hong Kong Special Administrative Region, China.

* Correspondence to: Dr. Linwei Tian, School of Public Health, Li Ka Shing Faculty of Medicine, The University of Hong Kong, 21 Sassoon Road, Pokfulam, Hong Kong. Phone: (+852) 2831 5071; Fax: (+852) 2855 9528; Email: linweit@hku.hk. This author takes responsibility for all aspects of the reliability and freedom from bias of the data presented and their discussed interpretation.

Funding support: This work was supported by the Health Medical Research Fund (Grant No. 11120311) and the Collaborative Research Award of the Faculty of Medicine, The Chinese University of Hong Kong (Grant No. CRA14-15/07).

\section{Contributor-ship statement:}

LWT, KFH and ITY defined the research theme. LWT, HQ and VCP analysed the data, interpreted the results and wrote the paper. CSC and KFH co-worked on associated data collection and their interpretation.

Conflict of Interest: The authors report no relationships that could be construed as a conflict of interest

Key Words: Carbon monoxide; Emergency Hospital Admission; Stroke; Time Series Study 


\section{Abbreviations:}

CO: carbon monoxide; DLM, distributed lag model; ER: Excess risk; HKEPD:

Environmental Protection Department of Hong Kong; IQR: interquartile range; $\mathrm{NO}_{2}$, nitrogen dioxide; $\mathrm{PM}_{2.5}$, particulate matter with aerodynamic diameter less than 2.5 microns; PACF, partial autocorrelation function; USEPA: Environmental Protection Agency of United States. 


\begin{abstract}
Background: Recent experimental and clinical studies suggested that exogenous carbon monoxide (CO) at low concentrations may have beneficial neuroprotective effects under certain circumstances. However, population-based epidemiological studies of environmentally relevant CO exposure generated mixed findings. The present study aimed to examine the short-term association of ambient CO with emergency stroke hospitalizations.
\end{abstract}

Methods: A time series study was conducted. Daily air pollution concentrations and emergency hospital admission data from January 2004 to December 2011 in Hong Kong were collected. Generalized additive Poisson models were used to estimate the associations between daily 24-hour mean concentrations of CO and emergency hospital admissions for stroke, while controlling for other traffic related co-pollutants: $\mathrm{NO}_{2}$ and $\mathrm{PM}_{2.5}$. Sensitivity analyses were performed using daily 1-hour maximum concentration of $\mathrm{CO}$ as exposure indicator.

Results: Negative associations were observed between ambient CO concentrations and emergency hospital admissions for stroke. The previous 1-3 day cumulative exposure to CO was associated with a $-2.0 \%(95 \% \mathrm{CI},-3.3 \%$ to $-0.7 \%)$ decrease in stroke admissions per interquartile range (IQR) increment in CO concentration (0.3ppm). Similar results were obtained when using 1-hour maximum concentration of CO as exposure indicator. The negative association was robust to the co-pollutant adjustment for either $\mathrm{NO}_{2}$ or $\mathrm{PM}_{2.5}$. Females and elders appeared to be more sensitive to ambient CO exposure. The negative association tended to be larger in cool season.

Conclusion: Short-term exposure to ambient CO was associated with decreased risk of emergency hospitalizations for stroke, suggesting some acute protective effects of CO exposure against stroke onsets. 


\section{Introduction}

Stroke was the second leading cause of death worldwide in 2011 and the fourth leading cause of death in Hong Kong, where more than 20,000 stroke patients per year were admitted to hospital for treatment and more than 3,000 of them died [1]. Many epidemiological studies linked short-term air pollution exposure to increased stroke onsets and emergency hospital admissions [2-7]. However, the particular effect of carbon monoxide (CO) on stroke was seldom reported. A few epidemiological studies that did examine the ambient CO on stroke admissions yielded mixed results. Several studies reported the significantly positive associations with $\mathrm{CO}$ and stroke [6-11], while others presented no associations with CO $[5,12-14]$.

A well-known toxicant, exogenous CO may also have physiologic and cytoprotective functions at low concentrations. Beneficial neuroprotective effects of $\mathrm{CO}$ under certain circumstances have been suggested by recent experimental and clinical studies [15-17]. Neuroprotection using exogenous CO as inhaled CO or injectable CO releasing molecules (CORM) is a novel and underexplored strategy [18]. Exogenous CO may control mitochondrial functioning, oxidative metabolism and substrate utilization, which changes neurometabolism and results in neuroprotection [19].

In the present study, we conducted a time series analysis to examine the association between the short-term variation of ambient CO concentrations and the risk of emergency stroke hospitalizations in Hong Kong. Given the strong role of traffic as a source for CO, we investigated whether associations between $\mathrm{CO}$ and stroke hospitalizations were robust to adjustment by other traffic-related pollutants $\mathrm{PM}_{2.5}$ or $\mathrm{NO}_{2}$. We also compared the seasonal difference, and did sub-group analyses by gender and age group, respectively, in order to capture the susceptible subpopulations. 


\section{Methods}

\subsection{Hospital Admission Data}

Daily counts of emergency hospital admissions for stroke, including all cerebrovascular diseases (ICD-9: 430-438) as the principal diagnosis from year 2004 to 2011 were obtained from the Hospital Authority Corporate Data Warehouse. Hospital Authority is the statutory body running all public hospitals in Hong Kong. The records of admission were taken from the publicly funded hospitals providing 24 hour accident and emergency services and covering $90 \%$ of hospital beds in Hong Kong for local residents [20]. We also computed the emergency stroke admissions by gender and by three age groups (age $<65,65 \sim 74$, and $>=75$ years old) for the subgroup analyses. Daily admissions for influenza (ICD-9:487) were used to identify influenza epidemics, which were then treated as a potential confounder in the regression models. Ethics approval and consent from individual subjects were not required by our institute as we used only aggregated data but not any individualized data in this study.

\subsection{Air Pollution and Meteorological Data}

Air pollution data between January 12004 and December 312011 were obtained from the Environmental Protection Department (EPD) of Hong Kong. There are 14 fixed monitoring stations including 11 general background stations and 3 roadside stations. The $\mathrm{CO}$ concentration data in this study were based on four general monitoring stations where CO records were available and represented the urban background pollution level. These background stations are Tsuen Wan (TW) and Yuen Long (YL) in the urban residential area, Tung Chung (TC) in the new town and Tap Mum (TM) in the rural area (Figure 1). Daily 24-hour mean concentrations across 4 general stations were calculated for CO and other traffic related pollutants $\mathrm{NO}_{2}$ and $\mathrm{PM}_{2.5}$ [21]. Sensitivity analyses were performed by using 
daily 1-hour maximum concentration of $\mathrm{CO}$ as exposure indicator. As Tap Mum station is located in remote area with only tens of residents, this site was excluded when calculating the city-wide average CO concentrations for some sensitivity analyses. Meteorological data on daily average temperature and relative humidity were obtained from the Hong Kong Observatory.

\subsection{Statistical modeling}

This was a longitudinal time series study. Generalized additive Poisson models (GAM) were used to fit the association between the daily $\mathrm{CO}$ concentrations and the emergency stroke hospitalizations. We used the smoothing spline, s(.), to filter out seasonal patterns and long-term trends in daily hospitalizations, as well as the daily mean temperature and relative humidity [22]. We also included an adjustment for the day of the week and dichotomous variables such as public holidays and influenza epidemic.

To reduce the problems associated with multiple testing and model selection strategies, we followed previous studies to select a priori model specification and degree of freedom $(d f)$ for the time trend and meteorological variables [21-23]. We used a $d f$ of 8 per year for the time trend, a $d f$ of 6 for the mean temperature of the current day $\left(\mathrm{Temp}_{0}\right)$ and the previous 3 days' moving average (Temp $\left.{ }_{1-3}\right)$ and a $d f$ of 3 for the current day relative humidity $\left(\right.$ Humid $\left._{0}\right)$. We included the day of the week (DOW) and public holidays (Holiday) in the model as dummy variables [24]. To adjust for the confounding effect of an influenza epidemic on emergency hospital admissions, we entered a dummy variable for the weeks with the number of influenza hospital admissions exceeding the 75 percentile in a year into the model [25].

Briefly, we set up a core model to remove the long term trends, seasonal variations, and adjust for time varying confounders as follows:

$\log (E(Y))=\alpha+s(t, d f=8 /$ year $\times$ no. of years $)+s\left(\right.$ Temp $\left._{0}, d f=6\right)+s\left(\right.$ Temp $\left._{1-3}, d f=6\right)+$ 
Here $E(Y)$ means the expected daily counts of emergency hospital admission for stroke on day $t$; $s($.$) is the smoothing spline function for nonlinear variables. We examined the residuals$ of the core model to check whether there were discernable patterns and autocorrelation by means of residual plot and partial autocorrelation function (PACF) plot. No discernible patterns and no autocorrelation in the residuals are the criteria for an adequate core model to control for time-varying confounding factors. The linear effect of CO was then estimated for the same day and up to three days prior to the outcome (single-lag effect from $\operatorname{lag}_{0}$ to $\operatorname{lag}_{3}$ ). The risk estimates for cumulative exposure to $\mathrm{CO}$ from the previous $1 \sim 2$ days $\left(\mathrm{dlm}_{12}\right)$ and 1 3 days $\left(\mathrm{dlm}_{13}\right)$ were fit by a unconstrained distributed lag model [26]. As traffic is one major source of $\mathrm{CO}$, we investigated whether associations between $\mathrm{CO}$ and stroke hospitalizations were sensitive to the adjustment for other traffic-related pollutants $\mathrm{PM}_{2.5}$ and $\mathrm{NO}_{2}$, respectively, in two-pollutant models where co-pollutants were included simultaneously with the same lag structure as CO. To justify the assumption of linearity between the logarithm of emergency stroke hospital admissions and CO, we graphically examined the exposure-response relationship using a smoothing function [27]. Sensitivity analyses were conducted to test the association by using the daily 1-hour maximum concentrations as the CO indicator. The 24-hour mean concentrations of CO averaged from 3 general stations excluding Tap Mum as exposure indicators were also performed in sensitivity analyses. Risk estimate differences across seasons, genders and age groups were also examined by using the subgroups of stroke hospitalizations as the health outcomes, respectively [28]. We tested the statistical significance of subgroup differences through calculating $\left(\beta_{1}-\beta_{2}\right) / \sqrt{S E_{1}{ }^{2}+S E_{2}{ }^{2}}$, where $\beta_{1}$ and $\beta_{2}$ are the estimates for the two categories (e.g., females and males), and $S E_{1}$ and $S E_{2}$ are their respective standard errors [28,29]. An absolute 
value larger than 1.96 was considered as statistically significant difference at $\alpha=0.05$ level.

The risk estimates were expressed in terms of the percentage changes (Excess Risk, ER (\%)) in emergency stroke hospital admissions per interquartile range (IQR) increment of CO, and their respective 95\% confidence intervals (CI). All analyses were conducted in the statistical environment R3.0.3 (R Development Core Team, 2014: http://www.r-project.org).

\section{Results}

\subsection{Data description}

During our study period, a total of 140,774 emergency hospital admissions for cerebrovascular diseases were recorded in our study population, accounting for about $27.7 \%$ of the total circulatory diseases. On average there were 48 emergency admissions per day for stroke, of which approximately $53.7 \%$ were males and $47.7 \%$ were elders with age $>=75$ years old. The daily mean admissions were slightly higher in cool season than in warm season (50 vs. 46 per day). The daily 24-hour mean concentration of CO was 0.65 ppm and the 1-hour maximum concentration of $\mathrm{CO}$ was $0.86 \mathrm{ppm}$, with the interquartile ranges (IQR) of $0.30 \mathrm{ppm}$ and $0.41 \mathrm{ppm}$ respectively. The daily mean concentrations of $\mathrm{PM}_{2.5}$ and $\mathrm{NO}_{2}$ were 37.0 and $45.6 \mu \mathrm{g} / \mathrm{m}^{3}$, respectively. CO concentrations were moderately correlated with both $\mathrm{NO}_{2}$ and $\mathrm{PM}_{2.5}$, with the correlation coefficients 0.63 and 0.67 , respectively. The daily mean air temperature was $23.4{ }^{\circ} \mathrm{C}$ and the relative humidity was $78 \%$. (Table- 1 )

\subsection{Regression results}

Negative associations were observed between ambient CO concentrations and daily emergency hospital admissions for stroke, after adjusting for the time trend, seasonality, meteorological factors, calendar effect and influenza epidemics. Every IQR (0.30 ppm) increase of CO at $\operatorname{lag}_{0} \sim \operatorname{lag}_{3}$ corresponded to $0.5 \sim 1.5 \%$ decrease of emergency hospital 
admissions for stroke. The previous 1 3 day cumulative exposure to $\mathrm{CO}\left(\mathrm{dlm}_{13}\right)$ was associated with $-2.0 \%$ (95\% CI, $-3.3 \%$ to $-0.7 \%$ ) decreases in stroke admissions per $0.3 \mathrm{ppm}$ increment of $\mathrm{CO}$ according to the single-pollutant model. Further adjustment for the possible confounding effects from traffic related co-pollutants at the same lags in two-pollutant models resulted in even stronger and statistically significant negative associations. Using the 1-hour maximum concentration of $\mathrm{CO}$ as exposure indicator produced similar results (Table-2). The results were not sensitive to the exclusion of Tap Mum, a remote air monitoring station, for calculating the city-wide average CO (not shown). The negative associations between $\mathrm{CO}$ and stroke admissions appeared to be stronger in females and elders: the gender difference was statistically significant on $\mathrm{dlm}_{12}$, with p-value of 0.046; the negative association in 'Age $>=75$ ' group was greater than that in 'Age of $65 \sim 74$ ' group on $\operatorname{lag}_{2}$, and $\mathrm{dlm}_{12}$, with p-values of 0.017 and 0.031 , respectively (Figure-2). The negative association appeared to be larger in cool season than in warm season, although statistical significance was not detected in either case which might relate to the reduced study power in sub-group analyses (Table-3).

Figure-3 shows the exposure-response curves for daily average $\mathrm{CO}$ at distributed lags of 1 3 days and risk of emergency hospital admissions for stroke in single pollutant models and two-pollutant models, after adjusting for the time trend, seasonality, meteorological factors, calendar effect and influenza epidemics. A smoothing spline with a $d f$ of 3 was applied on the CO concentration. The exposure-response curves were approximately linear at the ambient CO levels.

\section{Discussion}


This population-based epidemiological study demonstrated a negative association between ambient CO and hospital admissions for stroke. The negative association became stronger when the traffic-related co-pollutants $\mathrm{NO}_{2}$ and $\mathrm{PM}_{2.5}$ were adjusted for in two-pollutant models. Females and subjects older than 75 years appeared to be more sensitive to ambient CO.

Previous population-based epidemiological studies of environmentally relevant CO exposure and stroke hospitalizations generated mixed findings. Some studies indicated a negative association between $\mathrm{CO}$ and stroke hospitalizations but with no statistical significance $[5,30,31]$. Some other studies showing the adverse effects of CO on stroke did not adjust for the confounding effect of co-pollutants [13]. Positive associations between CO concentrations and stroke might not have persisted when adjusted by $\mathrm{NO}_{2}$ or PM. In a case-crossover study conducted in Copenhagen, Denmark [10], ambient CO was associated with an increased risk of ischemic stroke in single-pollutant models whereas the risk estimates for $\mathrm{CO}$ were attenuated to null when adjusting for PM. In Kaohsiung, Taiwan, significant positive associations were found between CO and all types of stroke; however, the risk estimates for CO turned statistically nonsignificant when either $\mathrm{NO}_{2}$ or $\mathrm{PM}_{10}$ was controlled for [4]. The lack of copollutant models has contributed to the inability to disentangle the effects attributed to CO from the larger complex air pollution mix (particularly motor vehicle emissions), and this creates uncertainty in interpreting the results observed in the epidemiological studies [32].

There were two recent systematic reviews and meta-analyses examining the short-term exposure to air pollution and stroke [6,7], and both reported the significantly positive associations between ambient CO exposure and the same day (lag0) stroke hospitalizations. Shah et al. acknowledged the publication bias according to the funnel plot and Egger's test for asymmetry [6,33]. According to the forest plot, Bell's study [11] assumed the greatest weight in Shah's review and meta-analysis. That was the largest multi-site time-series study conducted in 126 U.S urban counties which found positive and statistically significant association 
between same-day CO and increased risk of hospitalizations for cerebrovascular diseases. The association was attenuated but remained statistically significant with the traffic related co-pollutant adjustment [11]. For the lag1 and lag2 days’ CO exposure, however, Bell and colleagues reported negative associations with stroke hospitalizations. This was consistent with the current study that demonstrated negative associations for lag1 through lag3 days, in single-pollutant models and two-pollutant models that adjusted for $\mathrm{NO}_{2}$ or $\mathrm{PM}_{2.5}$. As the traffic was the main source for $\mathrm{CO}$, we considered the potential confounding effect by other traffic-related pollutants while examining the association of ambient CO with stroke hospitalizations. After adjustment for $\mathrm{NO}_{2}$, the negative associations of $\mathrm{CO}$ with stroke became stronger. Another study conducted in England and Wales [5] involving more than 460 thousand nationwide emergency stroke hospitalizations also observed a negative association between stroke and CO at lags 0-4 days. Although no statistical significance was detected, the effect size was comparable and consistent with what we observed in the current study. The negative association between ambient $\mathrm{CO}$ and stroke hospitalizations appeared to be stronger in females than in males. Instead of reflecting genetic susceptibility variability, the differentiated risk estimates across genders could result from different $\mathrm{CO}$ exposure profiles of males and females. Besides vehicle emissions, cigarette smoking serves another major source for CO exposure. The smoking rates were $3.0 \%$ in females and $19.9 \%$ in males in Hong Kong according to a survey in 2010 [34]. Chronic smokers exhibited carboxyhemoglobin levels of up to $9-12 \%$ whereas healthy subjects had levels between $1 \%$ and $2 \%$ of total blood volume $[35,36]$. For the smokers, the personal exposure to CO might be dominated by inhaled cigarette smoke [37] and thus a lack of correlation with ambient CO concentrations.

The short-term beneficial effects of ambient $\mathrm{CO}$ against stroke onsets are biologically plausible. Stroke is often associated with inflammation, ischemia reperfusion, or hemorrhagic compression. In all these manifestations, the role for hemo oxygenase (HO) and CO as major 
regulators has been indicated. The anti-inflammatory and beneficial neuroprotective effects of $\mathrm{CO}$ under certain circumstances have been suggested by recent experimental and clinical studies [15-17,38]. In ischemic stroke, the protective role of CO is mediated by the upregulation of Nuclear factor-erythroid 2-related factor 2 (Nrf2), a transcriptional factor that binds to response elements on the promoter region of the HO-1 gene [17]. In hemorrhagic stroke, CO alters the inflammatory response and reduce brain damage through a water-soluble carbon monoxide-releasing molecule 3 (CORM-3) [18]. However, the modulation of neurometabolic pathways by exogenous CO with resultant neuroprotection is likely dependent on the dose and timing of administration. Beneficial effects could be achieved when CORM-3 was given either before or 3 days after intracerebral hemorrhagic, that is, as a prophylactic agent or during the post-acute inflammatory phase [18]. We found stronger negative association between ambient $\mathrm{CO}$ and stroke hospitalizations in elders of age $>=75$ years. It is possible that the elderly may have more severe general inflammation milieu of the brain and lower cerebrovascular hemodynamics, thus more sensitive to the mediator role of CO. The negative association appeared to be larger in cool season than in warm season although statistical significance was not detected in either case which might relate to the reduced study power in sub-group analyses. Hong Kong is a subtropical city with very high prevalence of air conditioner usage in hot and humid summer but low prevalence of house heating in temperate winter. During the warm season (temperatures of $25^{\circ} \mathrm{C}-30^{\circ} \mathrm{C}$ and humidity of 71\%-91\% between 10th to 90th percentiles) people use air-conditioners more often and engage in less outdoor activities. But in the cool season in Hong Kong (temperatures of $14^{\circ} \mathrm{C}-24^{\circ} \mathrm{C}$ and humidity of $59 \%-90 \%$ between 10 th to 90 th percentiles), people are more likely to go outdoors and open the windows. Therefore, outdoor fixed-site air pollution measurement might better represent the average population exposure in cool season than in warm season in Hong Kong. 
The dose-response curves of the protective effects in stroke onset were approximately linear at the ambient levels of the 0.2 to $1.9 \mathrm{ppm}$, much lower than the CO levels administered in experimental investigations (around 20 250 ppm) [15]. However, for microenvironments that are in or near vehicles, the actual CO exposure levels can be much higher than the monitoring station measurements. It should also be noted that the exposure duration in the current time series epidemiology study was 24 hours a day (continuous exposure) while it has been usually one or two hours per day for experimental investigations. In fact, CO exposure duration and frequency are among the many unanswered questions for the application of inhaled $\mathrm{CO}$ as a therapeutic [15]. Examining the experimental data and the observational epidemiology data together may help to gain more insights into the biological effects of $\mathrm{CO}$ at environmentally relevant levels.

Given the ecological design of the present study, caution should be exercised in inferring cause-effect relations between low environmental CO exposure and stroke hospitalizations. Misclassifications in health outcomes and CO exposures were both likely. We used ICD-9 codes as the operational definition of stroke. These administrative codes might include a heterogeneous group of hospitalizations with differing risks, diagnoses and co-morbidities. Although the misclassification might not cause any differential bias, it warrants caution in the interpretation of negative associations between environmental CO and stroke. Merely modeling the associations between air pollution and health is not adequate because the association between an exposure and an outcome may not equal the causal effect, even after model adjustments for covariates. Even if a causal relationship is established, it is difficult to judge whether CO prevents the development or lessens the severity of stroke. Furthermore, we had access only to the aggregated data of overall stroke hospitalizations but not to individualized data with more specific diagnosis of stroke subtypes; we were not able to examine the potentially differentiated associations of $\mathrm{CO}$ with ischemic or hemorrhage stroke. 
Exposure misclassification was also likely because CO concentrations are spatially heterogeneous within a city and the small number of fixed monitoring stations might not be representative enough of the general population exposure. Based on aggregated measures of exposure and health outcomes, the time-series findings in the current study were subject to ecological fallacy. Moreover, the effect of prolonged CO exposure on stroke is unknown. The current time-series study design only allows us to infer about the acute effects but not the long-term effects which needs to be examined by experimental studies or epidemiological designs such as cohort or case-control studies.

In conclusion, we found low environmental CO associated with reduced risk of daily stroke hospitalizations, especially for females and elders. Given the ecological design of this study, caution should be exercised in inferring cause-effect relations. Even if the beneficial effects of ambient CO are confirmed for a segment of stroke patients, there may be detrimental effects on other subpopulations or other health endpoints, and there is the complication that $\mathrm{CO}$ is co-emitted with a number of other air pollutants. Findings on the short-term protective effects of CO on stroke hospitalizations need to be assessed together with other sources of evidence to better understand the health effects of CO at environmentally relevant levels.

\section{Acknowledgements:}

Authors thank the Environmental Protection Department of the Hong Kong Special Administrative Region for supplying the air monitoring data; Hospital Authority for providing the emergency hospital admission data and Hong Kong Observatory for providing weather data for this study. This work was supported by the Health Medical Research Fund (Grant No. 11120311) and the Collaborative Research Award of the Faculty of Medicine, The Chinese University of Hong Kong (Grant No. CRA14-15/07). 


\section{References:}

[1] P.H. Chau, J. Woo, W.B. Goggins, Y.K. Tse, K.C. Chan, S.V. Lo, et al., Trends in stroke incidence in Hong Kong differ by stroke subtype., Cerebrovasc. Dis. 31 (2011) 138-46. doi:10.1159/000321734.

[2] T. Yorifuji, E. Suzuki, S. Kashima, Cardiovascular Emergency Hospital Visits and Hourly Changes in Air Pollution., Stroke. 45 (2014) 1264-1268.

doi:10.1161/STROKEAHA.114.005227.

[3] G. a Wellenius, J. Schwartz, M. a Mittleman, Air pollution and hospital admissions for ischemic and hemorrhagic stroke among medicare beneficiaries., Stroke. 36 (2005) 2549-53. doi:10.1161/01.STR.0000189687.78760.47.

[4] S.-S. Tsai, W.B. Goggins, H.-F. Chiu, C.-Y. Yang, Evidence for an association between air pollution and daily stroke admissions in Kaohsiung, Taiwan., Stroke. 34 (2003) 2612-6. doi:10.1161/01.STR.0000095564.33543.64.

[5] A. Milojevic, P. Wilkinson, B. Armstrong, K. Bhaskaran, L. Smeeth, S. Hajat, Short-term effects of air pollution on a range of cardiovascular events in England and Wales: case-crossover analysis of the MINAP database, hospital admissions and mortality., Heart. 100 (2014) 1093-8. doi:10.1136/heartjnl-2013-304963.

[6] A. S. V. Shah, K.K. Lee, D. A. McAllister, A. Hunter, H. Nair, W. Whiteley, et al., Short term exposure to air pollution and stroke: systematic review and meta-analysis, Br. Med. J. 350 (2015) h1295. doi:10.1136/bmj.h1295.

[7] W.S. Yang, X. Wang, Q. Deng, W.Y. Fan, W.Y. Wang, An evidence-based appraisal of global association between air pollution and risk of stroke, Int. J. Cardiol. 175 (2014) 307-313. doi:10.1016/j.ijcard.2014.05.044.

[8] Y.-C. Hong, J.-T. Lee, H. Kim, H.-J. Kwon, Air Pollution: A New Risk Factor in Ischemic Stroke Mortality, Stroke. 33 (2002) 2165-2169. doi:10.1161/01.STR.0000026865.52610.5B.

[9] C.-C. Chan, K.-J. Chuang, L.-C. Chien, W.-J. Chen, W.-T. Chang, Urban air pollution and emergency admissions for cerebrovascular diseases in Taipei, Taiwan., Eur. Heart J. 27 (2006) 1238-44. doi:10.1093/eurheartj/ehi835. 
[10] Z.J. Andersen, T.S. Olsen, K.K. Andersen, S. Loft, M. Ketzel, O. Raaschou-Nielsen, Association between short-term exposure to ultrafine particles and hospital admissions for stroke in Copenhagen, Denmark., Eur. Heart J. 31 (2010) 2034-40. doi:10.1093/eurheartj/ehq188.

[11] M.L. Bell, R.D. Peng, F. Dominici, J.M. Samet, Emergency hospital admissions for cardiovascular diseases and ambient levels of carbon monoxide: results for 126 United States urban counties, 1999-2005., Circulation. 120 (2009) 949-55. doi:10.1161/CIRCULATIONAHA.109.851113.

[12] A.G. Barnett, G.M. Williams, J. Schwartz, T.L. Best, A.H. Neller, A.L. Petroeschevsky, et al., The Effects of Air Pollution on Hospitalizations for Cardiovascular Disease in Elderly People in Australian and New Zealand Cities, Environ. Health Perspect. 114 (2006) 1018-1023. doi:10.1289/ehp.8674.

[13] J. Kettunen, T. Lanki, P. Tiittanen, P.P. Aalto, T. Koskentalo, M. Kulmala, et al., Associations of fine and ultrafine particulate air pollution with stroke mortality in an area of low air pollution levels., Stroke. 38 (2007) 918-22. doi:10.1161/01.STR.0000257999.49706.3b.

[14] A. Tertre, S. Medina, E. Samoli, B. Fordberg, P. Michelozzi, A. Boumghar, et al., Short-term effects of particulate air pollution on cardiovascular diseases in eight European cities, J. Epidemiol. Community Heal. 56 (2002) 773-779.

[15] R. Motterlini, L.E. Otterbein, The therapeutic potential of carbon monoxide., Nat. Rev. Drug Discov. 9 (2010) 728-43. doi:10.1038/nrd3228.

[16] K.A. Hanafy, J. Oh, L.E. Otterbein, Carbon Monoxide and the Brain: Time to Rethink the Dogma, Curr. Pharm. Des. 19 (2013) 2771-2775.

[17] B. Wang, W. Cao, S. Biswal, S. Doré, Carbon monoxide-activated Nrf2 pathway leads to protection against permanent focal cerebral ischemia., Stroke. 42 (2011) 2605-10. doi:10.1161/STROKEAHA.110.607101.

[18] A. Yabluchanskiy, P. Sawle, S. Homer-Vanniasinkam, C.J. Green, R. Foresti, R. Motterlini, CORM-3, a carbon monoxide-releasing molecule, alters the inflammatory response and reduces brain damage in a rat model of hemorrhagic stroke., Crit. Care Med. 40 (2012) 544-52. doi:10.1097/CCM.0b013e31822f0d64. 
[19] C.S.F. Queiroga, A.S. Almeida, H.L. a Vieira, Carbon monoxide targeting mitochondria., Biochem. Res. Int. 2012 (2012) 749845. doi:10.1155/2012/749845.

[20] T.W. Wong, T.S. Lau, T.S. Yu, A. Neller, S.L. Wong, W. Tam, et al., Air pollution and hospital admissions for respiratory and cardiovascular diseases in Hong Kong, Occup Env. Med. 56 (1999) 679-83.

[21] H. Qiu, I.T.-S. Yu, X. Wang, L. Tian, L.A. Tse, T.W. Wong, Cool and dry weather enhances the effects of air pollution on emergency IHD hospital admissions., Int. J. Cardiol. 168 (2013) 500-5. doi:10.1016/j.ijcard.2012.09.199.

[22] R.D. Peng, F. Dominici, T. a. Louis, Model choice in time series studies of air pollution and mortality, J. R. Stat. Soc. Ser. A (Statistics Soc. 169 (2006) 179-203. doi:10.1111/j.1467-985X.2006.00410.x.

[23] F. Dominici, R. Peng, M. Bell, L. Pham, A. McDermott, S. Zeger, et al., Fine particulate air pollution and hospital admission for cardiovascular and respiratory diseases, JAMA (Journal Am. Med. Assoc. 295 (2006) 1127-1134. doi:10.1001/jama.295.10.1127.Fine.

[24] J. Schwartz, C. Spix, G. Touloumi, L. Bachárová, T. Barumamdzadeh, A. le Tertre, et al., Methodological issues in studies of air pollution and daily counts of deaths or hospital admissions., J. Epidemiol. Community Health. 50 Suppl 1 (1996) S3-11.

[25] C.-M. Wong, R.W. Atkinson, H.R. Anderson, A.J. Hedley, S. Ma, P.Y.-K. Chau, et al., A tale of two cities: effects of air pollution on hospital admissions in Hong Kong and London compared., Environ. Health Perspect. 110 (2002) 67-77.

[26] J. Schwartz, J.M. Samet, J. a. Patz, Hospital Admissions for Heart Disease: the effects of temperature and humidity, Epidemiology. 15 (2004) 755-761. doi:10.1097/01.ede.0000134875.15919.0f.

[27] C.-M. Wong, N. Vichit-Vadakan, H. Kan, Z. Qian, Public Health and Air Pollution in Asia (PAPA): a multicity study of short-term effects of air pollution on mortality., Environ. Health Perspect. 116 (2008) 1195-202. doi:10.1289/ehp.11257.

[28] H. Kan, S.J. London, G. Chen, Y. Zhang, G. Song, N. Zhao, et al., Season, sex, age, and education as modifiers of the effects of outdoor air pollution on daily mortality in Shanghai, China: The Public Health and Air Pollution in Asia (PAPA) Study., Environ. Health Perspect. 116 (2008) 1183-8. doi:10.1289/ehp.10851. 
[29] N. Schenker, J. Gentleman, On judging the significance of differences by examining the overlap between Confidence Intervals, Am. Stat. 5 (2001) 182-186.

[30] J.D. Poloniecki, R.W. Atkinson, a P. de Leon, H.R. Anderson, Daily time series for cardiovascular hospital admissions and previous day's air pollution in London, UK., Occup. Environ. Med. 54 (1997) 535-40.

[31] F. Ballester, J.M. Tenías, S. Pérez-Hoyos, Air pollution and emergency hospital admissions for cardiovascular diseases in Valencia, Spain., J. Epidemiol. Community Health. 55 (2001) 57-65.

[32] USEPA, Integrated Science Assessment for Carbon Monoxide (Full Report) (http://cfpub.epa.gov/ncea/cfm/recordisplay.cfm?deid=218686), (2010) (Accessed on 5 May 2014).

[33] M. Egger, G. Davey Smith, M. Schneider, C. Minder, Bias in meta-analysis detected by a simple, graphical test., BMJ. 315 (1997) 629-634.

doi:10.1136/bmj.316.7129.469.

[34] HKCSD, Thematic Household Survey Report No. 48, Hong Kong Census Stat. Dep. (2011) (Accessed on 8 May 2014). http://www.statistics.gov.hk/pub/B11302482011XXXXB0100.pdf.

[35] N. Wald, S. Howard, Letter:Variations in carboxyhaemoglobin levels in smokers, Br. Med. J. 1 (1975) 393.

[36] D. Castillo, P. Casan, Carbon monoxide: two sides to the same coin., Arch. Bronconeumol. 42 (2006) 489-91.

[37] E.T. Middleton, a H. Morice, Breath carbon monoxide as an indication of smoking habit., Chest. 117 (2000) 758-63.

[38] S.W. Ryter, A.M.K. Choi, Heme oxygenase-1/carbon monoxide: novel therapeutic strategies in critical care medicine, Curr. Drug Targets. 11 (2010) 1485-1494. 


\section{Figure legends:}

Figure-1 Map of Hong Kong showing the locations of the four general/background air monitoring stations (TW, TC, TM and YL) with carbon monoxide (CO) monitoring data.

Figure-2 Association of CO with Emergency Hospital Admissions for Stroke by Gender and Age Group. Risk estimates were expressed as Excess Risk (ER) with 95\%CI per IQR (0.3 ppm) increase of 24-hr mean concentration of CO. Unconstrained distributed lag models were used to estimate the ER associated with the previous 1 3 day cumulative exposure to $\mathrm{CO}$ ( $\mathrm{dlm}_{12}$ and $\mathrm{dlm}_{13}$ respectively). The gender difference was statistically significant on $\mathrm{d} \mathrm{lm}_{12}$, with p-value of 0.046 . The negative association in 'Age $>=75$ ' was greater than that in 'Age of 65 74'on lag, and dlm 12 , with p-values of 0.017 and 0.031 , respectively.

Figure-3 Exposure-response curves for daily mean concentration of $\mathrm{CO}$ at distributed lags of 1 3 days and its association with emergency hospital admissions for stroke in single-pollutant model and two-pollutant models, after adjusting for the time trend, seasonality, meteorological factors, calendar effect and influenza epidemics. The solid line represents the effect estimates; the dashed lines represent 95\% confidence intervals. 
Table-1 Distribution of Emergency Hospital Admissions for Stroke, Air Pollution

Concentrations and Meteorological Factors in Hong Kong, 2004-2011 (n=2922)

\begin{tabular}{|c|c|c|c|c|c|c|c|}
\hline & Mean & SD & Min. & $\mathbf{P}_{25}$ & $\mathbf{P}_{50}$ & $\mathbf{P}_{75}$ & Max. \\
\hline \multicolumn{8}{|l|}{$\begin{array}{l}\text { Emergency Hospital } \\
\text { Admissions (counts/day) }\end{array}$} \\
\hline All Circulatory Diseases & 173.8 & 30.0 & 97 & 152 & 170 & 192 & 319 \\
\hline Stroke & 48.2 & 7.9 & 22 & 43 & 48 & 53 & 78 \\
\hline In cool season & 50.2 & 7.8 & 28 & 45 & 50 & 55 & 78 \\
\hline In warm season & 46.2 & 7.5 & 22 & 41 & 46 & 51 & 73 \\
\hline Female & 22.3 & 5.1 & 6 & 19 & 22 & 26 & 42 \\
\hline Male & 25.9 & 5.5 & 7 & 22 & 26 & 29 & 51 \\
\hline Age $<65$ & 13.0 & 3.8 & 3 & 10 & 13 & 15 & 28 \\
\hline Age of $65 \sim 74$ & 12.2 & 3.8 & 1 & 10 & 12 & 15 & 27 \\
\hline Age $>=75$ & 23.0 & 5.4 & 7 & 19 & 23 & 26 & 50 \\
\hline \multicolumn{8}{|l|}{$\begin{array}{l}\text { Air pollution } \\
\text { concentrations }\end{array}$} \\
\hline CO (24-hr mean, ppm) & 0.65 & 0.23 & 0.23 & 0.47 & 0.60 & 0.77 & 1.94 \\
\hline CO (1-hr Max., ppm) & 0.86 & 0.33 & 0.30 & 0.62 & 0.80 & 1.03 & 3.03 \\
\hline $\mathrm{PM}_{2.5}\left(\mu \mathrm{g} / \mathrm{m}^{3}\right)$ & 36.97 & 21.86 & 6.15 & 18.59 & 33.34 & 49.86 & 143.40 \\
\hline $\mathrm{NO}_{2}\left(\mu \mathrm{g} / \mathrm{m}^{3}\right)$ & 45.59 & 17.95 & 8.55 & 31.96 & 43.02 & 56.49 & 138.72 \\
\hline \multicolumn{8}{|l|}{ Meteorological factors } \\
\hline Mean temperature $\left({ }^{\circ} \mathrm{C}\right)$ & 23.4 & 5.2 & 8.8 & 19.3 & 24.6 & 27.8 & 31.8 \\
\hline Humidity (\%) & 77.9 & 10.5 & 31.0 & 73.0 & 79.0 & 85.0 & 98.0 \\
\hline
\end{tabular}

Abbreviation: SD, standard deviation; $\mathrm{P}_{\mathrm{x}}, \mathrm{x}^{\text {th }}$ percentiles; Min., minimum; Max., maximum. 
Table-2 Percent Change (ER\% (95\% CI)) of Emergency Hospital Admissions for Stroke per IQR Increment of CO by lags, 2004-2011 ${ }^{\text {a }}$

\begin{tabular}{|c|c|c|c|c|c|c|}
\hline Pollutant & $\mathbf{L a g}_{0}$ & $\mathbf{L a g}_{1}$ & $\mathbf{L a g}_{2}$ & $\mathbf{L a g}_{3}$ & $\operatorname{dlm}_{12}{ }^{b}$ & $\mathbf{d l m}_{13}{ }^{\mathrm{c}}$ \\
\hline \multicolumn{7}{|c|}{ ngle-pollutant model } \\
\hline CO (24-hr mean) & $-0.5(-1.5,0.5)$ & $-1.5(-2.5,-0.5)$ & $-1.3(-2.4,-0.3)$ & $-1.0(-2.0,-0.0)$ & $-1.8(-2.9,-0.6)$ & $-2.0(-3.3,-0.7)$ \\
\hline CO (1-hr Max.) & $0.1(-0.8,1.0)$ & $-1.1(-2.0,-0.2)$ & $-0.9(-1.8,-0.0)$ & $-0.7(-1.6,0.2)$ & $-1.4(-2.4,-0.3)$ & $-1.6(-2.8,-0.4)$ \\
\hline \multicolumn{7}{|c|}{ vo-pollutant model ${ }^{\mathrm{d}}$} \\
\hline $\mathrm{CO}$ & $-0.5(-1.8,0.8)$ & $-1.1(-2.4,0.2)$ & $-1.5(-2.8,-0.3)$ & $-1.7(-2.9,-0.4)$ & $-1.7(-3.1,-0.2)$ & $-2.1(-3.6,-0.5)$ \\
\hline $\mathbf{P M}_{2.5}$ & $0.0(-1.4,1.5)$ & $-0.6(-2.0,0.7)$ & $0.4(-0.9,1.7)$ & $1.2(-0.1,2.5)$ & $-0.2(-1.7,1.2)$ & $0.3(-1.3,2.0)$ \\
\hline \multicolumn{7}{|c|}{ vo-pollutant model ${ }^{\mathrm{d}}$} \\
\hline $\mathrm{CO}$ & $-1.9(-3.1,-0.6)$ & $-1.8(-3.1,-0.6)$ & $-2.2(-3.4,-1.0)$ & $-2.1(-3.3,-0.9)$ & $-2.6(-4.0,-1.3)$ & $-3.1(-4.6,-1.6)$ \\
\hline $\mathrm{NO}_{2}$ & $2.4(1.1,3.7)$ & $0.6(-0.6,1.9)$ & $1.5(0.3,2.7)$ & $1.9(0.7,3.1)$ & $1.3(-0.1,2.7)$ & $2.0(0.4,3.6)$ \\
\hline
\end{tabular}

\footnotetext{
a: The interquartile ranges (IQRs) were $0.30 \mathrm{ppm}$ and $0.41 \mathrm{ppm}$ for CO of 24-hr mean and 1-hr maximum concentrations, respectively; the IQRs were 31.27 and $24.53 \mu \mathrm{g} / \mathrm{m}^{3}$ for $\mathrm{PM}_{2.5}$ and $\mathrm{NO}_{2}$, respectively. b: previous 1-2 day cumulative exposure to CO. ${ }^{\text {c }}$ : previous 1-3 day cumulative exposure to CO.

d: 24-hour mean concentration of pollutants were used in two-pollutant models.
} 
Table-3 Percent Change (ER\% (95\% CI)) of Emergency Hospital Admissions for Stroke per IQR Increment of CO by lags in Cool and Warm Seasons Respectively, 2004-2011 a

\begin{tabular}{|c|c|c|c|c|c|c|}
\hline Season & $\operatorname{Lag}_{0}$ & $\mathbf{L a g}_{1}$ & $\mathbf{L a g}_{2}$ & $\operatorname{Lag}_{3}$ & $\operatorname{dlm}_{12} b$ & $\operatorname{dlm}_{13}{ }^{c}$ \\
\hline \multicolumn{7}{|l|}{ Cool Season } \\
\hline CO only & $-0.3(-1.5,0.9)$ & $-1.4(-2.6,-0.2)$ & $-1.4(-2.6,-0.2)$ & $-1.4(-2.6,-0.2)^{*}$ & $-1.8(-3.2,-0.4)$ & $-2.3(-3.8,-0.7)$ \\
\hline $\mathrm{CO}$ adj. for $\mathrm{PM}_{2.5}$ & $-0.3(-1.8,1.1)$ & $-1.3(-2.7,0.1)$ & $-1.5(-2.9,-0.1)$ & $-1.8(-3.2,-0.5)$ & $-1.8(-3.3,-0.2)$ & $-2.4(-4.1,-0.6)^{*}$ \\
\hline $\mathrm{CO}$ adj. for $\mathrm{NO}_{2}$ & $-1.6(-3.0,-0.2)$ & $-1.9(-3.3,-0.5)$ & $-2.0(-3.3,-0.6)$ & $-2.1(-3.5,-0.8)$ & $-2.5(-4.0,-1.0)$ & $-3.0(-4.7,-1.3)$ \\
\hline \multicolumn{7}{|l|}{ Warm Season } \\
\hline CO only & $-0.3(-2.2,1.7)$ & $-1.4(-3.3,0.5)$ & $-1.1(-3.0,0.8)$ & $0.3(-1.6,2.2)$ & $-1.5(-3.6,0.6)$ & $-0.9(-3.3,1.6)$ \\
\hline $\mathrm{CO}$ adj. for $\mathrm{PM}_{2.5}$ & $-0.3(-3.2,2.8)$ & $0.4(-2.5,3.5)$ & $-0.8(-3.7,2.2)$ & $0.1(-2.8,3.1)$ & $-0.2(-3.5,3.1)$ & $0.4(-3.3,4.1)$ \\
\hline $\mathrm{CO}$ adj. for $\mathrm{NO}_{2}$ & $-2.3(-5.0,0.4)$ & $-1.8(-4.6,1.0)$ & $-2.7(-5.5,0.1)$ & $-1.5(-4.3,1.3)$ & $-3.1(-6.2,0.0)$ & $-3.1(-6.5,0.5)$ \\
\hline
\end{tabular}

a: 24-hr mean concentration of CO was used in the model, with the interquartile range (IQR) $0.30 \mathrm{ppm}$. ${ }^{\mathbf{b}}$ : previous 1-2 day cumulative exposure to CO. ${ }^{\mathbf{c}}$ : previous $1-3$ day cumulative exposure to CO. ${ }^{*}$ : Compared with warm season at the same lag and in the same single or two-pollutant model, $\mathrm{p}<0.10$. 